\title{
Protein Expression Analysis in Hematopoietic Stem Cells during Osteopontin-Induced Differentiation of Natural Killer Cells
}

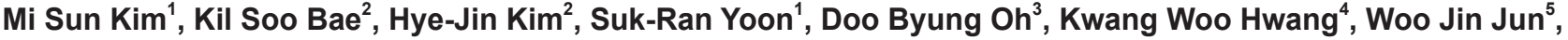 \\ Sang In Shim ${ }^{6}$, Kwang-Dong Kim ${ }^{7}$, Yong-Woo Jung ${ }^{8}$, So-Young Park ${ }^{9}$, Ki Sun Kwon ${ }^{3}$, Inpyo Choi ${ }^{1, *}$ \\ and Jin Woong Chung ${ }^{2, *}$
}

${ }^{1}$ Cell Therapy Research Center, Korea Research Institute of Bioscience and Biotechnology, Daejon 305-806,

${ }^{2}$ Department of Biological Science, Dong-A University, Busan 604-714,

${ }^{3}$ Aging Research Center, Korea Research Institute of Bioscience and Biotechnology, Daejon 305-806,

${ }^{4}$ Department of Immunology, College of Pharmacy, Chung Ang University, Seoul 156-756,

${ }^{5}$ Department of Food and Nutrition, Chonnam National University, Gwangju 500-757,

${ }^{6}$ Department of Agronomy, Gyeongsang National University, Jinju 660-701,

${ }^{7}$ Division of Applied Life Science (BK21), Gyeongsang National University, Jinju 660-701,

${ }^{8}$ Department of Immunology, College of Pharmacy, Korea University Sejong Campus, Yeongi-gun 339-700,

${ }^{9}$ Department of Nanobiomedical Science, Dankook University, Chungnam 330-714, Republic of Korea

\begin{abstract}
Natural Killer (NK) cells are the lymphocytes that are derived from hematopoietic stem cells, developed in the bone marrow from hematopoietic stem cells (HSC) by sequential acquisition of functional surface receptors, and express the repertoire of inhibitory and activating receptors. Recently, Osteopontin (OPN) has been identified as a critical factor for differentiation of natural killer cells. However, the detailed mechanism of OPN-induced NK differentiation has been still to be elucidated. Here, we determined the signaling pathway and possible receptor for OPN in NK differentiation. OPN induced expression of Bcl-2 and activation of Erk kinase. Inhibition of Erk pathway decreased the effect of OPN on NK differentiation. In addition, the expression of integrin $\alpha 9$ was significantly increased by OPN during NK differentiation, suggesting the possible role of a major signaling molecule for OPN- induced NK differentiation.
\end{abstract}

Key Words: Osteopontin, Natural killer cells, Hematopoietic stem cells, Erk, Bcl-2

\section{INTRODUCTION}

NK (NK) cells are the lymphocytes that are derived from hematopoietic stem cells in the bone marrow. NK cells play a crucial role in innate immunity and tumor surveillance by eliminating transformed cells as well as virus-infected cells upon activation. These effector functions are performed by the release of cytokines, such as IFN- $\gamma$, GM-SCF, and TNF- $\alpha$ (Perussia, 1996). NK cells are developed in the bone marrow from hematopoietic stem cells (HSC) by sequential acquisition of functional surface receptors (Di Santo, 2006; Freud and Caligiuri, 2006). Many factors are involved in the process of NK maturation which includes cytokines, surface proteins and transcription factors as well as microenvironmental factors in bone marrow. Recently, we identified osteopontin (OPN) as a critical factor for natural killer cell differentiation from HSC (Chung et al., 2008). OPN is a secreted protein which is mainly expressed in bone marrow stromal cells. OPN has been known to play a variety of roles in cellular mechanisms including proliferation, apoptosis, and migration. In addition, OPN has been reported to act as a negative regulator of HSC although the effect of OPN on HSC proliferation or apoptosis is controversial. For example, it was shown that OPN reduces the number of HSCs by inhibition of proliferation while increasing apoptosis (Stier et al., 2005). On the contrary, it has been reported that OPN negatively regulates HSC proliferation in- www.biomolther.org

Open Access DOI: 10.4062/biomolther.2011.19.2.206

pISSN: 1976-9148 elSSN: 2005-4483

Copyright $\odot 2011$ The Korean Society of Applied Pharmacology
Received Dec 6, 2010 Revised Mar 9, 2011 Accepted Mar 14, 201

*Corresponding Author

E-mail: ipchoi@kribb.re.kr (Choi IP), jwchung@dau.ac.kr (Chung JW)

Tel: +82-42-860-4223 (Choi IP), +82-51-200-7270 (Chung JW)

Fax: +82-42-860-4220 (Choi IP), +82-51-200-7269 (Chung JW) 
dependently on induction of apoptosis (Nilsson et al., 2005). Thus, to further dissect the mechanisms of OPN-induced NK differentiation, we investigated the gene expression profiles and signaling pathways in HSC during OPN-induced NK development.

\section{MATERIALS AND METHODS}

\section{In vitro differentiation of NK cells}

NK differentiation from HPC was performed as previously described (Chung et al., 2008). Briefly, c-Kit ${ }^{+}$, lineage-negative $\left(\mathrm{Lin}^{-}\right)$(B cells [B220], T/NK cells [CD2], granulocytes [Gr-1], monocytes [CD11b], NK/NKT cells [NK1.1] and erythrocytes [TER-119]-depleted) HSC were purified by the magnetic cell sorting (MACS) Cell Separation kit (Miltenyi Biotec, Bergisch Gladbach, Germany) according to the manufacturer's protocol. Antibodies used for MACS purification were purchased from Becton Dickinson and BD Pharmingen. The purified HSC were plated onto 24-well plates (Becton Dickinson) at $1 \times 10^{6}$ cells per well and cultured in RPMI medium supplemented with a mixture of stem cell factor (SCF) $(30 \mathrm{ng} / \mathrm{ml}$; Bio-Source, Camarillo, CA), Flt3L (50 ng/ml; Peprotech, Rocky Hill, NJ), IL-7 (0.5 ng/ml; Peprotech), indometacin $(2 \mu \mathrm{g} / \mathrm{ml}$; Sigma-Aldrich, St. Louis), and gentamicin $(20 \mu \mathrm{g} / \mathrm{ml})$ for 6 days at $37^{\circ} \mathrm{C}$, $5 \% \mathrm{CO}_{2}$. The culture medium was refreshed every 3 days, and recombinant osteopontin (rOPN) was added to the culture on the 3rd day as needed. To generate the mature NK (mNK) cells, the cells were then cultured with OP9 stromal cells (a gift from Dr. T. Nakano, University of Osaka, Suita, Japan) in the presence of IL-15 (50 ng/ml; Peprotech). After 4-6 additional days of culture, the number of NK1.1+ cells was determined via flow cytometric analysis using anti-CD122 antibody (BD, Franklin Lakes, NJ).

\section{Western blot analysis}

Cells were lysed in lysis buffer (20 mM HEPES ( $\mathrm{pH} 7.9$ ), $100 \mathrm{mM} \mathrm{KCl}, 300 \mathrm{mM} \mathrm{NaCl}, 10 \mathrm{mM}$ EDTA, 1\% Triton X-100, $0.5 \mathrm{mM}$ PMSF, and protease inhibitors mixture [Roche]) for 20 min on ice, and then centrifuged at $13,000 \mathrm{~g}$ for $20 \mathrm{~min}$. The supernatants were heat denatured in the presence of 2-ME and SDS, and separated electrophoretically in a 10\% SDSpolyacrylamide gel under denaturing conditions. The proteins were then transferred electrophoretically onto a polyvinylidene difluoride membrane (Millipore), and the membrane was incubated sequentially with a primary $\mathrm{Ab}$ and a HRP-conjugated anti-mouse or anti-rabbit IgG secondary Ab (Amersham Biosciences). The protein bands were visualized using ECL Western blot detection reagents (Amersham Biosciences) on X-ray film (Sigma-Aldrich).

\section{FACS analysis}

All antibodies except for flow cytometric analysis were purchased from Becton Dickinson (Franklin Lakes, NJ) and BD Pharmingen (San Diego), and cells from individual tissues were stained with the indicated antibodies in a staining buffer (phosphate-buffered saline [PBS] containing 1\% fetal bovine serum [FBS] and $0.01 \% \mathrm{NaN} 3$ ) for 20 minutes at $4^{\circ} \mathrm{C}$.

\section{Quantitative real time RT-PCR}

For RT-PCR, SYBR Premix Ex Taq (Takara Bio) was used to detect OPN expression with a Dice TP 800 Thermal Cycler
A

Control

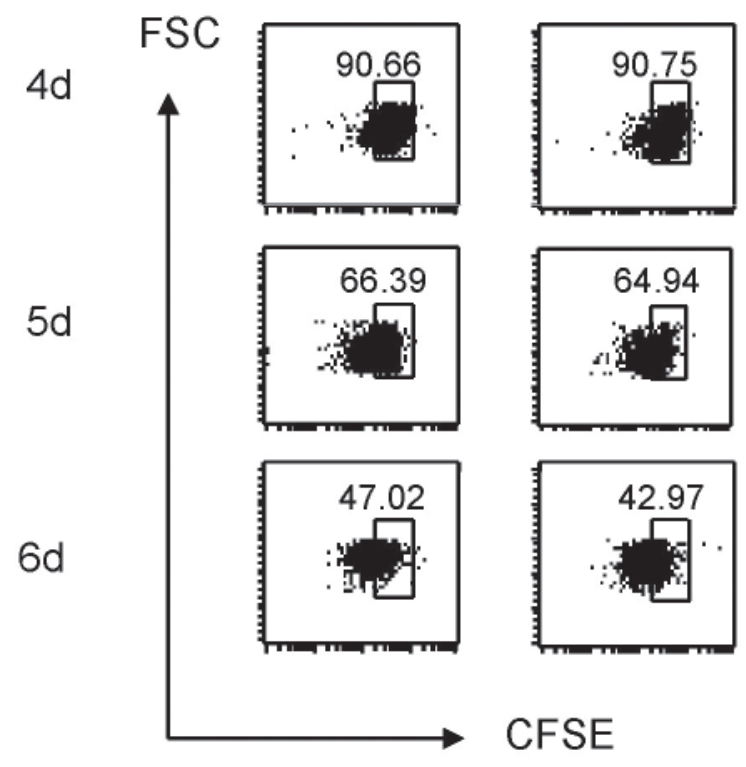

B

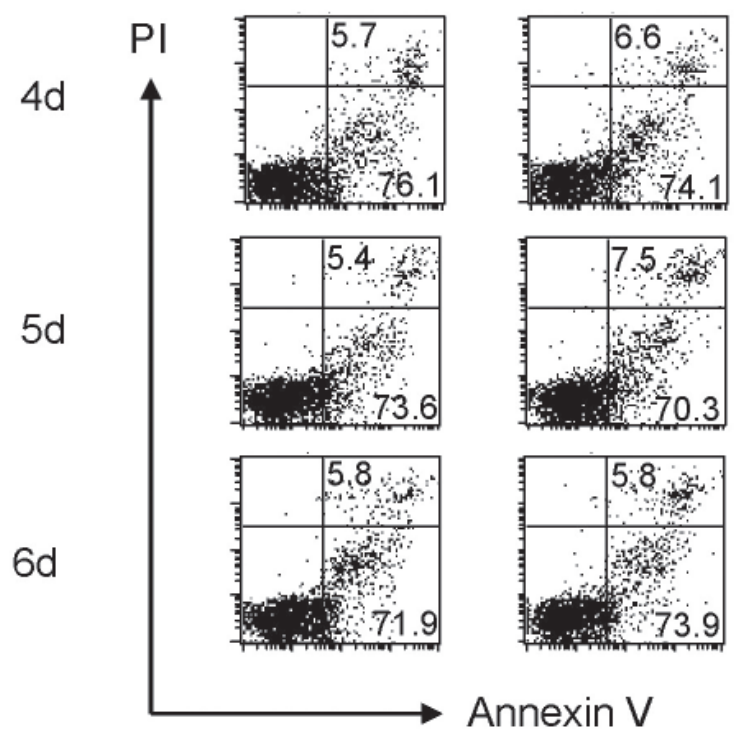

Fig. 1. Effect of OPN on survival and proliferation of NK cells. NK cells were differentiated from HSC in vitro with or without rOPN (2 $\mu \mathrm{g} / \mathrm{ml})$ as described in Materials and Method. HSCs were stained with CFSE for proliferation assay (A), and with PI and annexin $\mathrm{V}$ for analysis of apoptosis (B), and subject to FACS analysis.

(Takara Bio). The primers used were as follows: CD122, 5'-ACAGGCCCAAGATTCAGTCCAC-3' and 5'-CCTCAAGCCTGACCTGATCCA-3'; Integrin $\beta 1$, 5'-TCCCGACATCATCCCAATTGTA3 ' and 5'- CACAGTTGTCACGGCACTCTTGTA-3'; Integrin $\beta 3$, 5'-TTCAATGCCACCTGCCTCAA-3' and 5'-CCTTGGCCTCGATACTAAAGCTCA-3'; Integrin $\alpha$ v, 5'-GCTGTGGACCGAGA CGTTCA-3' and 5'-ATCCTCGATTGGCAGGTTCTTG-3'; Integrin $\alpha 4,5^{\prime}$-GCTGGCGATGATGCTTACGA-3' and 5'-GCTGCAGGCAAGCTTCACTA-3'; Integrin a9, 5'-CACTGATACAT- 


\section{Con OPN2 OPN5}

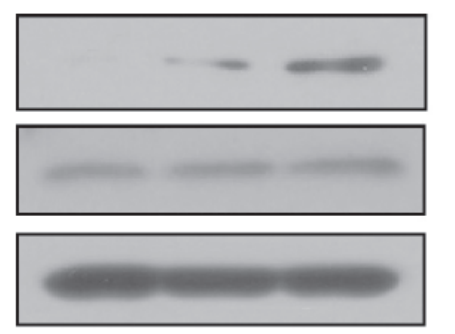

$\mathrm{Bcl}-2$

$\mathrm{Bcl}-\mathrm{xL}$

$\beta$-actin

Fig. 2. Expression of anti-apoptotic proteins during OPN-induced NK differentiation. After 6 days of in vitro NK differentiation from HSCs in the absence or presence of indicated concentrations of OPN (con; control, opn2; $2 \mu \mathrm{g} / \mathrm{ml}$, opn5; $5 \mu \mathrm{g} / \mathrm{ml}$ ), cells were lysed in sample buffer containing $2 \%$ SDS. Proteins in whole lysates (100 $\mu \mathrm{g} / \mathrm{ml}$ ) were resolved by SDS/PAGE, electroblotted onto PVDF membranes, and probed with antibodies specific for $\mathrm{Bcl}-2$ and $\mathrm{Bcl}-$ $\mathrm{xL}$. $\beta$-acin was included as a loading control.

GCAGCAGGC-3' and 5'-TCCTGGGCTCTGAACTCTGA-3'; CD44, 5'-CCAATGGGACAAGGTCATCA-3' and 5'-ATTTGGAGCTGCAGTAGGCT-3'; $\beta$-actin, 5'-AGGCCCAGAGCAAG AGAGG-3' and 5'-TACATGGCTGGGGTGTTGAA-3'. The value for the expression of each target gene was calculated as the relative quantity of each gene divided by the relative quantity of actin.

\section{Statistical analysis}

All experiments were repeated at least three times. Data are presented as means \pm standard deviation. A student's $t$ test was used to compare means and $p<0.05$ was considered as significant.

\section{RESULTS}

\section{Effect of OPN on survival and proliferation of NK cells}

OPN has been well known as an anti-apoptotic protein or even an oncoprotein in a variety of cell types (Metge et al., 2010). However, previous study reported that OPN is a negative regulator of HSC proliferation in vivo (Nilsson et al., 2005). According to the reports, however, naïve OPN did not affect the proliferation of HSC in vitro while thrombin-cleaved OPN exhibited inhibition of proliferation or survival of HSC. Thus to confirm whether OPN affects HSC proliferation under circumstances that direct NK differentiation, we investigated the effect of OPN on proliferation of HSC during NK differentiation in vitro. The results showed that administration of recombinant OPN did not affect the in vitro proliferation of HSC compared to the control (Fig. 1A). In addition, OPN also did not affect the apoptosis of HSC during in vitro NK differentiation (Fig. 1B). Taken together, these results showed that naïve OPN did not affect either proliferation or survival of HSCs during NK differentiation in vitro, while it enhances HSC differentiation towards NK cells.

\section{Expression of anti-apoptotic proteins during OPN-in- duced NK differentiation}

We next investigated whether OPN affect the expressions of anti-apoptotic proteins via Western blot analysis. Interest-

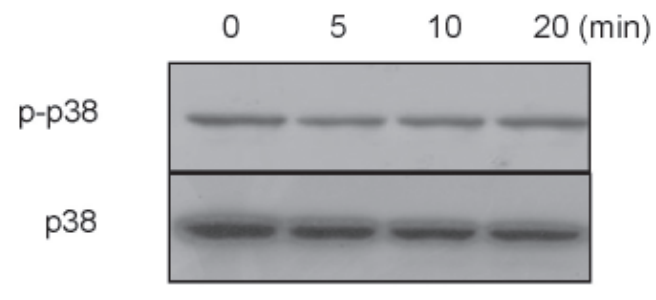

p-Erk

Erk

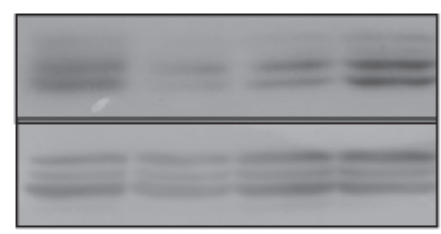

Fig. 3. Activation of MAP kinases. On the $6^{\text {th }}$ day of in vitro NK differentiation, cells were lysed and subject to Western blot analysis. The activation levels of p38 and Erk were monitored by immunoblot with specific antibodies to phosphorylated form of each MAP kinase.

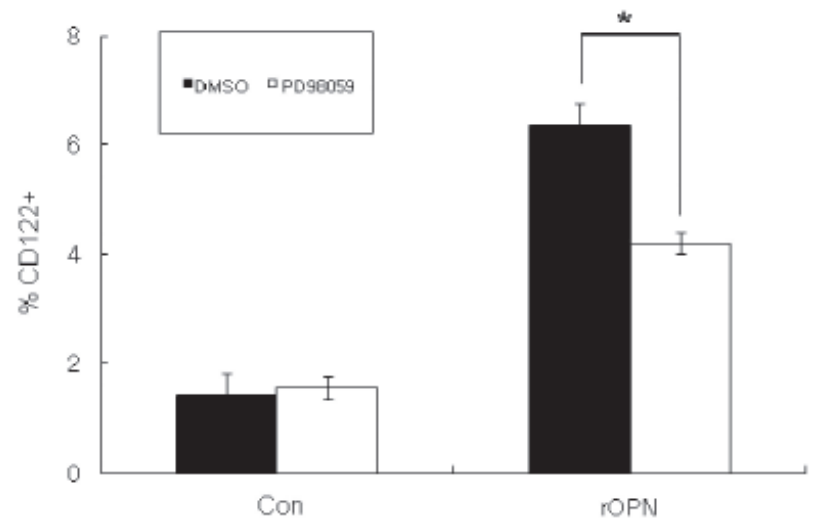

Fig. 4. Effect of OPN on Erk activation for NK differentiaiton. Erk activation was blocked by pretreatment of PD98059 $(10 \mu \mathrm{M})$ for 30 minutes prior to adimistration of rOPN on 3 day HSCs. Effect of Erk inhibition on OPN-induced NK differentiation was measured by FACS analysis. Data are expressed as a percentage of the CD122-positive population, and represent the mean \pm SD of triplicate determinations $\left({ }^{*} p<0.05\right)$.

ingly, OPN induced expression of $\mathrm{Bcl}-2$ in HSCs in a dose dependent manner while Bcl-xL was not significantly affected by OPN (Fig. 2). Since OPN did not affect the survival of HSC in the previous experiment, the increased expression of $\mathrm{Bcl}-2$ by OPN may play a role in differentiation of NK cells rather than the survival of HSC itself.

\section{Activation of MAP kinases}

Various MAP kinases have been known to be involved in proliferation, survival, and differentiation of a variety of cell types. Thus, we next investigated whether OPN affects the activation of MAP kinases such as p38 and Erk during NK differentiation. Western blot analysis showed that OPN increases activation of Erk while it did not affect the activation of p38 (Fig. 3), suggesting that Erk activation may be involved 


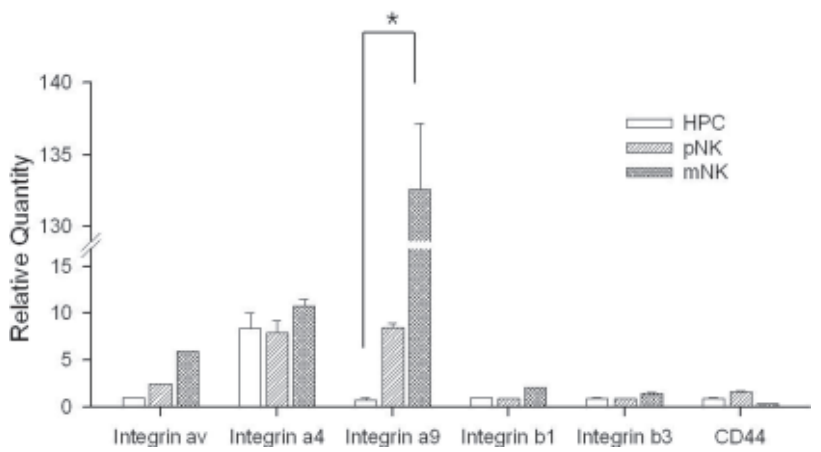

Fig. 5. Expression of Osteopontin Receptors in HSC during in vitro differentiation of NK cells. Expressions of various subunits of integrin recpetors $(\alpha \mathrm{V}, \alpha 4, \alpha 9, \beta 1, \beta 3$ indicated as av, a4, a9, b1 and $b 3$, respectively) and CD44 during OPN-induced NK differentation were analyzed by quantitative RT-PCR. HPC: hematopoietic precursor cells, pNK: precursor NK cells, mNK: mature NK cells. Data are representative of four individual experiments $\left({ }^{*} p<0.005\right)$.

in OPN-induced NK differentiation. Indeed, blockage of Erk activation by administration of PD98059, an inhibitor of Erk, decreased the population of NK precursors $\left(C D 122^{+}\right)$as determined by FACS analysis (Fig. 4), proving the involvement of Erk activation in OPN-induced NK differentiation.

\section{Expression of osteopontin receptors in HSC during in vitro differentiation towards NK cells}

Several integrins and CD44 are known as cellular receptors for OPN in various mechanisms depending on the cell types (El-Tanani, 2008; Orian-Rousseau, 2010). Thus, to investigate the changes in receptor expression, we analyzed the expression levels of various integrin receptors and CD44 during OPN-induced differentiation of HSC. Quantitative RTPCR showed that expression of integrin $\alpha 9$ was prominently increased during in vitro differentiation of NK cells (Fig. 5), suggesting that integrin $\alpha 9$ may act as a major receptor of OPN for NK differentiation in HSC.

\section{DISCUSSION}

Previously, we identified OPN as a critical factor for NK cell differentiation (Chung et al., 2008). However, T-bet is the only factor that has been known to be involved in OPN-induced NK differentiation. Thus, the detailed mechanisms of NK differentiation by OPN still remain to be elucidated. Here, we have analyzed the expression profiles of various proteins during OPN-induced NK cell differentiation. Since the role of OPN in survival of HSC is controversial, we first investigated whether OPN affects the apoptosis of HSC during NK cell differentiation, and found that OPN did not significantly affect the cell death or proliferation of HSC, at least in the culture condition toward NK differentiation. Interestingly, although OPN does not seem to be involved in apoptosis of HSC, it induces the expression of $\mathrm{Bcl}-2$, which is a representative of the anti-apoptotic proteins. Thus, the induction of $\mathrm{Bcl}-2$ by OPN may play a role in NK differentiation itself rather than survival of HSC or NK cells. Indeed, Bcl-2 induction by IL-21, which promotes the differentiation of NK cells, has previously been demonstrated in T cells and NK cells (Bonanno et al., 2009; Ostiguy et al.,
2007). Bcl-2 has been known to activate several MAP kinases such as p38 and Erk in a variety of cell types (Mauchera et al., 1998; Sheridan et al., 2010). In fact, our results showed that OPN induced activation of Erk, but not of p38 during NK differentiation, suggesting that Erk pathway is critical for NK differentiation induced by OPN. Several receptors for osteopontin have been identified in several cell types for a variety of physiological mechanisms, such as proliferation, differentiation and chemotaxis. For example, $\alpha$ X $\beta 2$ integrin functions as an OPN receptor for chemotaxis in monocytes (Schack et al., 2009), while OPN mediates metastasis via integrin $\alpha V \beta 3$ and CD44 in tumor cells (Robertson et al., 2010). In this study, we found that expression of integrin $\alpha 9$ is notably increased in HSC by treatment of OPN. This result suggests its role as a major factor that initiates signaling events in OPN-induced NK cell differentiation. Taken together, our results show that OPN induces expression of $\mathrm{Bcl}-2$ and activation of Erk, possibly via interaction with integrin $\alpha 9$, leading to the promotion of NK differentiation.

\section{ACKNOWLEDGMENTS}

This work was supported by a grant from Korea Research Council of Fundamental Science and Technology (KRCF) project, Republic of Korea.

\section{REFERENCES}

Bonanno, G., Mariotti, A., Procoli, A., Corallo, M., Scambia, G., Pierelli, L. and Rutella, S. (2009) Interleukin-21 induces the differentiation of human umbilical cord blood CD34-lineage- cells into pseudomature lytic NK cells. BMC Immunol. 10, 46.

Chung, J. W., Kim, M. S., Piao, Z. H., Jeong, M., Yoon, S. R., Shin, N., Kim, S. Y., Hwang, E. S., Yang, Y., Lee, Y. H., Kim, Y. S. and Choi, I. (2008) Osteopontin promotes the development of natural killer cells from hematopoietic precursor cells. Stem Cells 26, 21142123

Di Santo, J. P. (2006) Natural killer cell developmental pathways: a question of balance. Annu. Rev. Immunol. 24, 257-286.

El-Tanani, M. K. (2008) Role of osteopontin in cellular signaling and metastatic phenotype. Front Biosci. 1, 4276-4284.

Freud, A. G. and Caligiuri, M. A. (2006) Human natural killer cell development. Immunol. Rev. 214, 56-72.

Mauchera, C., Weissingerb, E. M., Kremmerc, E., Baccarinind, M., Procykd, K., Hendersone, D. W., Wolffe, L., Kolcha, W., Kaspersf, B., Mushinskie, J. F. and Mischaka H. (1998) Activation of bcl-2 suppressible 40 and $44 \mathrm{kDa}$ p38-like kinases during apoptosis of early and late B lymphocytic cell lines. FEBS Letter. 427, 29-35

Metge, B. J., Liu, S., Riker, A. I., Fodstad, O., Samant, R. S. and Shevde, L. A. (2010) Elevated osteopontin levels in metastatic melanoma correlate with epigenetic silencing of breast cancer metastasis suppressor 1 . Oncology 78, 75-86.

Nilsson, S. K., Johnston, H. M., Whitty, G. A., Williams, B, Webb, R. J., Denhardt, D. T., Bertoncello, I, Bendall, L. J., Simmons, P. J. and Haylock, D. N. (2005) Osteopontin, a key component of the hematopoietic stem cell niche and regulator of primitive hematopoietic progenitor cells. Blood 106, 1232-1239.

Orian-Rousseau, V. (2010) CD44, a therapeutic target for metastasising tumours. Eur. J. Cancer 46, 1271-1277.

Ostiguy, V., Allard, E. L., Marquis, M., Leignadier, J. and Labrecque, N. (2007) IL-21 promotes T lymphocyte survival by activating the phosphatidylinositol-3 kinase signaling cascade. J. Leukoc. Biol. 82, 645-656.

Perussia, B. (1996) The cytokine profile of resting and activated NK cells. Methods 9, 370-378. 
Robertson, B. W., Bonsal, L. and Chellaiah, M. A. (2010) Regulation of Erk1/2 activation by osteopontin in PC3 human prostate cancer cells, Molecular Cancer 9, 260.

Schack, L., Stapulionis, R., Christensen, B., Kofod-Olsen, E., Skov Sørensen, U. B., Vorup-Jensen, T., Sørensen, E. S. and Höllsberg, P. (2009) Osteopontin enhances phagocytosis through a novel osteopontin receptor, the alphaXbeta2 integrin, J Immunol. 182 6943-6950.

Sheridan, C., Brumatti, G., Elgendy, M., Brunet, M. and Martin, S. J (2010) An ERK-dependent pathway to Noxa expression regulates apoptosis by platinum-based chemotherapeutic drugs. Oncogene. 29, 6428-6441.

Stier, S., Ko, Y., Forkert, R., Lutz, C., Neuhaus, T., Grünewald, E., Cheng, T., Dombkowski, D., Calvi, L. M., Rittling, S. R. and Scadden D. T. (2005) Osteopontin is a hematopoietic stem cell niche component that negatively regulates stem cell pool size. J. Exp. Med. 201, 1781-1791.

Véronique Orian-Rousseau. (2010) CD44, a therapeutic target for metastasising tumours. Eur. J. Cancer 46, 1271-1277. 